\title{
Proposal for the creation of a Web-based heterogeneous distributed archive for psychological data
}

\author{
STEVEN M. BOKER and CHARLES R. CROWELL \\ University of Notre Dame, Notre Dame, Indiana
}

\begin{abstract}
This report presents a proposal to create archives of data from psychological research and associated metadata Web pages and link them into a heterogeneous distributed archive on the World-Wide Web. Several specific recommendations are made concerning some of the issues faced by the data archivist and data archive user hoping to use the Web. In particular, a recommendation is made to create a publicly accessible Web page for each data set and place keywords, experimental methods, data descriptions, pointers to journal articles, and pointers to other archive Web pages pertinent to this data set on this metadata Web page. If the archivist includes a special keyword (PsychologyDataArchive) on the metadata Web page, Web-based search engines will automatically be able to subset all participating data archives for indexing and semantic analysis. The secondary data analyst can then include the word PsychologyDataArchive in his Web search and will be able to effectively find relevant participating Web data archives.
\end{abstract}

The Internet began its life as a network serving research scientists and has since grown to encompass most of the networked computers on the planet today. Services on the Internet were primarily limited to file transfer protocol (FTP), remote terminal (Telnet), and e-mail until the advent of the World-Wide Web (WWW, or the Web) in 1991. The Web grew slowly for the first few years of its existence but, since then, has grown at an exponential rate. The Web has changed the criteria by which the limitations of computing are defined. The combined storage capacity and computing power of all of the machines connected to the Web is truly enormous. There are an estimated 171 million plus computers attached to the Internet (Internet Software Consortium, 2003) over 45 million Web sites (Netcraft, 2003), and over 3 billion documents indexed by several major search engines (Sullivan, 2003). Data archiving has become an issue of increasing interest to scientific societies and funding agencies (see Kurzman, Church, \& Crystal, 2002, for a recent overview). This article is intended to further the discussion about archiving by proposing an intermediate step between centralized archives maintained by societies or agencies and independent disconnected archives maintained by individual researchers.

This article is based in part on a presentation given by the first author at the Symposium on Data Archiving in Psychology, American Psychological Association Annual Convention, Chicago, August 16, 1997. Funding for this work was provided, in part, by NIH Grant 1R29 AG14983. The authors acknowledge the help of the editor and four reviewers in improving and sharpening the ideas presented herein. Correspondence may be addressed to S. M. Boker, Department of Psychology, University of Notre Dame, Notre Dame, IN 46556 (e-mail: sboker@nd.edu; see also http://www.nd.edu/ sboker).

\section{Some Recent History}

Although the process of clicking links in a browser is by now familiar to almost everyone, it is useful to consider how the transfer of the browser's focus from one page to the next is accomplished. Web pages are connected through the universal resource locator (URL) mechanism that allows the links from one page to call pages on machines locally or on remote computers around the world without the user needing to know where each Web page physically resides. In addition, if the page to which a link points is changed, as long as the URL stays the same, the calling page is not affected. The worst that can happen is a so-called broken link when a URL disappears - a mild annoyance, but of no more import than a dead-end street.

Web pages are written in a text-based format, using a subset of standard generalized markup language (SGML). This subset is called the hypertext markup language (HTML) and was the initial standard that evolved for creating Web-based content. The basis for HTML is a system of coding alphanumeric characters called $A S C I I$ (American standard code for information interchange). ASCII is by far the most widely used and enduring standard in computing - at the core of the Web and basically unchanged since it was adopted by the American National Standards Institute (ANSI) in 1963.

HTML employs tags inserted into the ASCII text in order to create text formatting, as well as to handle placement of graphics and hypertext links. These links not only can send the user to another page, but also can perform calls to programs that run on the Web server or provide a simple mechanism for the user to download information. In recent years, many advances have occurred in HTML itself, as well as in systems that support and extend it on both the client side (i.e., a user's local machine) 
and the server side (i.e., the Web server). These enhancements include the following: the emergence of the extensible markup language (XML), which represents a kind of metalanguage with which users can overcome many of the limitations inherent in HTML; client-side languages, such as Java and JavaScript, that are designed to run inside the user's local Web browser, providing additional programming functionality; and server-side scripting languages, such as active server pages (ASP) and hypertext preprogramming (PHP).

\section{The Web and Semantic Distance}

As a result of these developments, the Web has redefined how distance is represented in our access to informationwith respect to both the obvious change in the physical distance walked to the library and the semantic distance between meaningful objects. For example, with the Web, it is not necessary to construct or utilize elaborate physical taxonomies, such as the Dewey decimal system, to identify and store information. As users of conventional libraries well know, the Dewey decimal system places books with similar subjects near to each other on a library shelf, thereby equating physical distance with semantic distance. However, this is a one-dimensional information structure; that is, any given book can be next to only two other books on the shelf. In contrast, on the Web, the information-indexing structure can be multidimensional; thus, many Web pages may be next to each other. To the extent that pages with similar semantic content link to one another, the semantic distance between pages can be measured as a function of the network of links (i.e., ASCII HTML tags within the text) between them. This means that the Web has the ability to represent associational or semantic distance directly as part of the textual information itself.

Within this structure, so-called Web harvesters (also called intelligent agents, or spiders) began to emerge that were designed to search through the Web automatically for the purposes of retrieving and indexing the material they found. Harvesters have contributed greatly to the usefulness of the Web as a place where information can be found quickly and easily. As they became more sophisticated, various harvesters have been able to search and index all nonpassword-protected pages on the Web, thereby enabling powerful search engines, such as Google, Alta Vista, and Ask Jeeves.

Google, in particular, has become the dominant search engine for the Web. Google's basic premise is to organize its index of pages by using the implicit semantic distance inherent in the links between pages. Not only does this strategy indicate which pages are important (in that their URL appears on many other pages), but also it yields an index of semantic nearness that provides a listing of pages more likely to be related to the search topic than would a simple search for words on a page.

The present article proposes a distributed solution to some of the problems associated with behavioral science data archiving. Other solutions include centralized sci- entific society sponsored archives, such as that being instituted by the Psychonomic Society. We expect that both distributed and centralized archives will find their place in scientific research. Although many of the issues in the remainder of the article pertain to both centralized and distributed archives, we will focus primarily on the special strengths and problems associated with distributed archives, where individual research labs retain responsibility for the storage and dissemination of their data.

\section{DATA ARCHIVING: WHAT AND WHY}

This section will briefly address two questions: (1) What is data archiving? and (2) Why do it? A data archive is a repository for information gathered during the course of scientific research. By definition, empirically based scientific research yields some form of raw data derived from systematic observations. An archive represents a place where that accumulated data is stored.

Different types of scientific research produce different kinds of data, depending on the nature of the subject matter being investigated and the types of observational processes employed. In psychological research, the data generally are related to what organisms say or do (i.e., their behavior) but also could include quantitative or qualitative measures of internal states, physiological processes, or other properties. Although the data gathered in psychological research often are numerical in nature, they can take many other forms, including text, sounds, graphics, still pictures, motion video, or some combination of these media. A data archive for psychological research thus needs to be able to store information in any or all of these forms.

To be maximally useful, data archives should be available to other researchers. This criterion gets at the why of data archiving. The main purpose of a data archive is to facilitate the sharing of information among researchers. Since science is a collective endeavor involving simultaneous research by different investigators in different locations, the ability to share findings and results is of the utmost importance. Although journals and other forms of scholarly publication enable such sharing to occur at a certain level, page space is never sufficient to include all the necessary details of any particular investigation, especially the raw data involved. An archive, then, would enable other scientists to access and examine data they did not (or could not) collect and to offer their own secondary analyses as supplements to what was provided by the primary researcher. In this way, the "openness" and collective nature of scientific research is enhanced, as is the potential for teaching and training new scientists.

Encoding the raw data from scientific investigations in electronic form makes an archive easier to manage and to access. Electronic formats permit dissemination and access over networks or by means of other easily distributable media (e.g., CDs or DVDs). The Internet and the WWW are perhaps the ultimate archiving tools in this respect. 


\section{THE BASIC QUESTIONS FOR DATA ARCHIVING}

There are two vantage points from which to view the data-archiving process: the perspective of the data analyst and that of the data archivist. These differing standpoints are for the most part complementary, although in some instances they may be at odds with one another. Important questions about the archiving process arise from both standpoints. The purpose of this section is to identify and define these questions. Further commentary related to these matters will be presented in later sections.

\section{Questions From the Data Analyst's Perspective}

We here will identify five central questions of interest to the data analyst, the user of a data archive. Although we will consider these questions in the context of a proposal for a distributed archive, these questions can be pertinent for users of centralized archives as well.

How does one find the variables of interest within an archive? This issue will be a central problem if the data are to be distributed among a wide variety of archive sites across the Web. In order to find the proper variables for secondary analysis, much must be known concerning the experimental conditions under which the data were gathered, as well as how the variables were coded and stored. Information about data is called metadata. Metadata needs to be stored as part of any archive in order to allow the data itself to be retrievable and useful once retrieved. Metadata should include information both in human-readable and machine-readable format, so that both human-directed and automatic searches can be performed. It is critical that the metadata itself be trivially easy to access, since otherwise one could be trapped in an infinite regress of needing metadata for the metadata. One possible format that suggests itself is embedding the metadata into HTML so that Web browsers can view it and Web harvesters can index it.

How does one obtain permission to use the data from an archive? The data store may contain confidential or limited access materials. The prospective secondary analyst must be able to contact the archivist if permission needs to be granted to gain access to the data. Although raw data may require access restrictions, the metadata for that raw data is likely not to require restricted access. Thus, indexable metadata Web pages could be used as access restriction mechanisms. Since Web pages can include e-mail links, a convenient method for establishing contact with the archivist is provided.

How does one query the archive to obtain the data? Sometimes, only a few variables or a subset of individuals' records within a larger data archive are of interest to the secondary analyst. This process of selecting a subset of the data can involve a limiting query directed at the archive itself or a query executed locally after downloading the entire data set. A balance between convenience for the data analyst and work for the data archivist must be struck. Very large public use databases, such as the United States cen- sus data, provide both methods: a Web-based point-andclick query for subsets and tabular information, as well as full access to all of the data tables in ASCII format. It seems unlikely that Web-based query systems will become widespread for distributed scientific data archives, since there is the risk that if the work for the data archivist is too great, the archive would not be instituted at all.

How does one combine data from different archives? In order to join the same variable from different archives, statistical techniques, such as multitrait-multimethod (Marsh \& Grayson, 1995; Rafaeli \& Tractinsky, 1991) and incomplete data structural equation (McArdle \& Hamagami, 1992; McArdle \& Horn, 1985) models can be used to take advantage of the maximum information available. In order to join different variables from several data archives, it is necessary to have one or more common index variables that can serve to link the data records together. This linking can be quite powerful but presents problems of its own, such as increased danger of loss of subject confidentiality. We will address some of the confidentiality issues due to linking in a later section.

How does one analyze the data? Secondary analysis presents its own set of benefits and challenges that must be carefully considered. The secondary analysis of data can involve a variety of techniques and is a broad topic that exceeds the scope of the present article.

\section{Questions From the Data Archivist's Perspective}

We next will identify five questions of importance to the data archivist, the researcher or team responsible for making data accessible in a distributed archive. The issues that pertain to centralized archives follow the same general categories, but many of the specific concerns are unique to the distributed archivist.

How does one store data in an archive? Some format must be chosen for data storage. This format should be readable by the maximum number of researchers and should still be able to be read in 50 or 100 years. For these reasons, many data archivists have been choosing to store their data in rectangular arrays as ASCII text characters. Although this format is less efficient than others, it guarantees the maximum portability and durability, since proprietary database formats have the tendency to change as the software goes through revisions. When the data are images, audio, or video, we recommend choosing formats that are (1) widely used and (2) openly documented. It is an unfortunate fact of the computer software industry that proprietary formats often remain secret and undergo revisions in order to keep users of commercial software from purchasing alternatives. Although widely used openly documented formats may also change, these formats are likely to have openly available translation programs to allow migration between one format and another. It is not in the interest of a commercial software company to provide a migration path away from their proprietary format. Data migration is a nonignorable issue for data archives, since computing platforms evolve rapidly. It could be cata- 
strophic to have a data archive locked into the proprietary format of a software company that goes out of business; 10, 20, or 50 years later, a computer that can run the software necessary to read the archive will be increasingly difficult to find.

How does one store the metadata about an archive? As was noted above, the metadata consists of descriptions of the variables, how the data is coded and stored, and the methods used to perform the experiment that generated the data. This information is essential if the secondary data analyst is to use the data effectively. If these data are kept in an ASCII-readable format, such as HTML, they can be processed with a variety of searching, indexing, and associational tools now in wide use on the Web. We recommend that each data set in an archive have a metadata Web page. Certainly, keywords are an obvious form of metadata that should be included on this Web page. Another form of metadata is the journal article in which the results of the analysis of the archived data set are described. As such, a link to a text-searchable form of that article would be a crucial part of a distributed archive metadata Web page. In addition, source code in an opensource language such as R (Ihaka \& Gentleman, 1996) could be provided as a means for reading the data. Opensource languages may change over time but are likely to provide migration paths to many computer platforms and operating systems, thereby reducing the possibility of having the source code become obsolete or be usable only on an obsolete computer. Finally, semantic distance to other research in the field can be added by linking a metadata page to other distributed archive pages deemed relevant by the archivist or users. Adding these links not only helps secondary researchers navigate between nodes of the distributed archive network, but also can give search engines such as Google the raw material needed to build semantic networks between archive nodes.

How does one provide confidentiality and control access to the archive? Data may include confidential information that needs to be removed or encrypted prior to archiving. Sometimes, confidential portions of these data may not be able to be completely removed, in which case access to the data must be restricted. Even when access is not restricted, archivists may wish to track the use of the data for purposes of justifying their archiving efforts to funding agencies. At the same time, it is in the interest of the archivist to provide metadata in the most unrestricted way. Access to journal articles may be restricted by publishers, and so the journal article as metadata may need to be subject to a reprint request from a researcher. It is unclear to the authors whether this fair use reprint request mechanism applies to requests from automatic search engines. Such an interpretation of fair use would be of benefit to the scientific community, since semantic networks could be built linking metadata described in peer-reviewed print journals.

How does one link to other, similar data sets in other archives? Providing data that can be linked to other data sets, particularly longitudinal studies in which the link- ing variable must be an individual identifier, can pose difficult questions regarding confidentiality. Public key encryption techniques may be able to allow secondary analysts to link between data archives without knowing the identity of the individual subjects. In such a technique, each archive would have a public and a private key, so that individual data records could be linked only when both archives participated in the link. This would require a software system running at both participating archives that would perform the encrypted link for a third-party data analyst. Although this is theoretically possible to achieve in an automatic fashion, it is likely that such links would be achieved only given the active involvement of the archivists at each participating distributed site. Linking is likely to be more easily achieved with centralized archives than with distributed archives.

How does one advertise the availability of an archive? One measure of the success of a data archive is when the data are used for novel and important analyses that the original researcher had not foreseen. Often, this means that the existence of the data must be made known to scholars who are not in the same field as the original researcher. This requires a form of broadcast advertisement that can come to the notice of potential users even when the archivist does not know who or where those potential users may be. Web search agents can help potential users of the data find variables of interest if the archivist gives the search agents the raw material needed to construct an effective index. Well-constructed metadata Web pages will both serve to advertise the availability of the data and maximize its usefulness, once discovered by the secondary analyst.

\section{A SUCCESS STORY: GENBANK}

The field of molecular genetics has produced a spectacular success story in data archiving, the GenBank Project (GenBank, 1997). GenBank is the NIH-sponsored, publicly accessible record of almost all known DNA sequences maintained by the National Center for Biotechnology Information. There are millions of DNA sequences recorded in GenBank, and the system has been tailored so as to be accessible over the Web.

GenBank has been remarkably successful in archiving and indexing data from the molecular genetics community. This success is due to two reasons: (1) The major molecular genetics journals came to an agreement that all articles submitted for publication must first deposit their sequence data in GenBank and receive a tracking number, and (2) the highly effective indexing and similarity-matching software has made the archive an important part of a great many secondary analyses.

GenBank has created a unique system in which the bibliographic information, keyword indexing, and DNA sequence data are all interlinked, as is shown in Figure 1. Both the DNA sequence data and the keyword data are associated using an algorithm that calculates a distance between each data point and its neighbors. In addition, the 


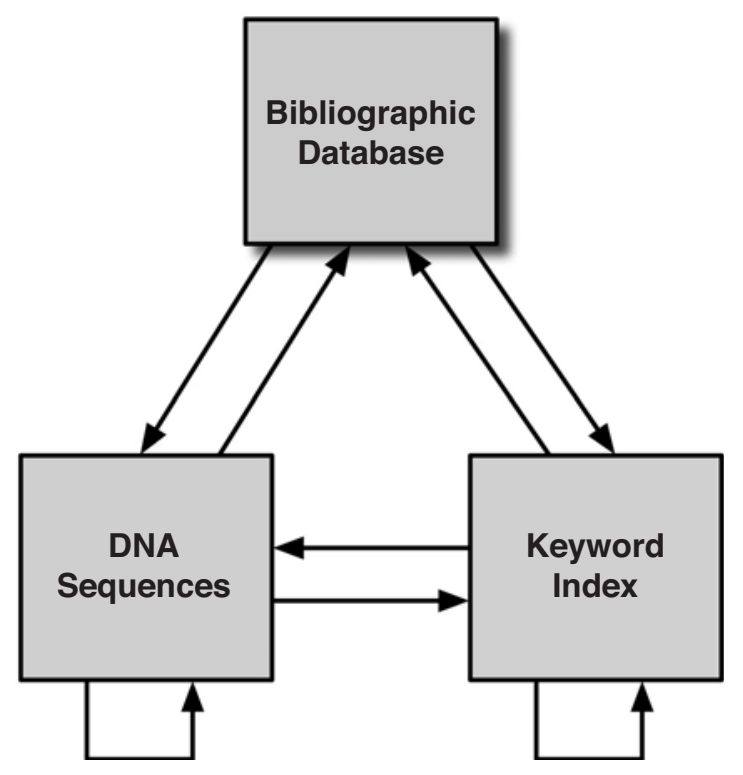

Figure 1. The organization of the three parts of GenBank.

bibliographic, keyword, and DNA sequence data are all cross-referenced with respect to molecular neighborhoods.

This means that a secondary analyst can search in a variety of ways. For instance, one researcher might look up a keyword and find that it produces several bibliographic matches, each of which point to a DNA sequence. Those DNA sequences might be nearly like another sequence that then points back to a new keyword and bibliographic references. To illustrate, Koutarou, Tissenbaum, Liu, and Ruvkun (1997) reported a surprising finding in which a gene that is associated with longevity in the worm Caenorhabditis elegans was found to be similar to the gene for the insulin receptor in humans. This finding may have major consequences for the study of diabetes, and its discovery may have been long delayed without the aid of GenBank.

\section{HETEROGENEOUS DISTRIBUTED DATA}

Unfortunately, psychological data are not so neat and well constrained as the base pair sequences that make up DNA sequences. Psychological data can take a variety of forms, as was noted above, including categorical or continuous numerical tables or matrices, mixed alphanumeric and numeric tables, tables with relations among them, natural language text, images, and video and audio data. Each of these data types can have many possible storage formats, and each of the storage formats can have many possible query formats, leading to a scientific Tower of Babel.

In order to ensure that the data in an archive is readable by the maximum number of users for the maximum length of time, it is important to choose data storage and query formats that are (1) simple to implement, (2) open and nonproprietary, and (3) in wide use for a relatively long period of time. For data in table or matrix format, we recommend that the data be archived as ASCII-formatted text files with fixed-width columns separated by space characters. Although this format is not the most convenient for use from any one computer platform, it is the most likely to be able to be read by all computer platforms and the most likely to still be readable 50 or 100 years from now.

Images and video and audio files present different problems. Digital pictures can be encoded in a variety of formats, and there is no single standard. Among the most popular picture formats today are PDF and JPEG, but there are many others. JPEG is the most common image format in use on the Web and so is likely to be stable for a relatively long time and, when changed, is likely to have a migration path. PDF is becoming a standard for the interchange of scientific documents, although it is a proprietary format and sometimes produces inconsistent viewing results on different computing platforms, depending on which software version created the PDF document and which software version is viewing it. We consider it to be too soon to tell whether PDF will become a stable standard that will still be widely available in 50 years.

Digital audio has been very popular on audio CDs for some time now, and the 16-bit linear format used on CDs is commonly used for digitizing stereo audio sound. However, the MP3 format for compressed audio storage has become the de facto Web standard. MP3 files have the advantage of being widely available and audible on most computing platforms. In addition, MP3 files do not have digital rights management (DRM) copy protection mechanisms built into them. It is self-defeating to archive data that would have a mechanism that would prevent it from being copied or transferred to a new format. We recommend that the data archivist steer clear of any data format with a built-in copy protection mechanism.

Digital video formats are in flux. There are several competing self-declared "standards," and digital video requires so much storage space that it is nearly always stored in a compressed format (e.g., MPEG). In the home entertainment market, the DVD format using MPEG 2 compression has emerged as a dominant standard. On the Web, formats such as Apple's QuickTime, Microsoft's Windows Media Format, and Real Software's Real Video all have become popular, but as of yet there is no clear single Web-based standard format for motion video. In addition, each of these Web formats is proprietary, and all of them share the previously mentioned problems associated with commercial proprietary formats.

Once the data format is chosen, the data archivist must decide how the metadata is to be stored. We recommend that the metadata be stored as ASCII text embedded in an HTML file that comprises a metadata Web page describing experimental methods, variables, and storage mechanisms for each data set in the data archive. We recommend that the journal article or articles that have analyzed the data set be linked from the metadata Web 
page. In addition, we recommend that links to other, similar metadata Web archive pages be included.

To review, the demands of heterogeneous distributed data archiving on the Web require that the data archivist (1) make the format of the data as simple as possible, (2) provide clear explicit descriptions of how the data are stored, (3) provide clear explicit descriptions of the meaning of the variables, (4) provide an abstract, a method section, and a pointer to published articles, and (5) link to similar metadata archive pages.

\section{DATA RETRIEVAL ISSUES}

After the data format has been chosen, there still remains the question of how delivery of the data is to be accomplished. The interested secondary data analyst will most likely need only a subset of the data in the archive. Two main possibilities exist for addressing this need for querying and subsetting. Either the secondary analyst can transfer the entire data set to his local machine and then perform the query, or a query mechanism can be embedded within the archive itself, so that the query can be performed prior to delivery of the data.

Each system has its advantages and disadvantages. Retrieving the entire database and querying locally has the disadvantages that (1) the data storage is duplicated on both the local and the remote machines, (2) network bandwidth is required to transfer the full data set, and (3) increased effort on the part of the data analyst is required in order to perform the query locally. However, retrieving the entire database has the advantage that the data archivist does not need to provide a method for local query, which can involve a considerable expense of time and computing resources.

Providing an embedded query mechanism requires the archivist to use one of the systems available for managing Web queries from on-line databases (e.g., MySQL, PostgreSQL, or Oracle). Some sites, such as the University of Virginia's Geospatial and Statistical Data Center, have used a combination of Perl and CGI scripts with a Web interface to perform queries on SAS databases. These methods have the advantage of being easier to use for the data analyst, and they minimize the amount of network traffic. These considerations can be substantial when the archived database is very large (e.g., U.S. census data).

When the data to be archived is less than $100 \mathrm{MB}$ in size or is likely to be used by only a few secondary analysts, we recommend that the data archive deliver the entire data set. Here, archivists should explore the potential of XML to store and deliver data to users in formats readily compatible with standard applications, such as spreadsheets. However, when there is likely to be a large number of users and the data set is large, we recommend that the archivists consider budgeting for the programming required to provide a easy-to-use Web-based query process or utilize available Web-enabled systems, using Oracle, PostgreSQL, or MySQL, that have built-in query tools.

\section{DATA-JOINING ISSUES}

Some secondary analysis projects require joining records from different databases. For instance, individual records might be linked to community level variables from the U.S. census, or two sets of individual records might be linked together to provide longitudinal measurement information. There are a special set of problems inherent in data joining; some of these problems are technical, and some concern issues in subject confidentiality.

In order to join two databases, one must have an acceptable index key that will identify which record is to be matched to which. This index key could be a variable such as ZIP code when linking community-level variables or could be a subject identifier when linking records by subject. Of course, if two databases use different randomized subject IDs, these variables become useless as an index key to link the two databases. For this reason, there must be some coordination between the data archivists whose databases are involved in a data-joining project.

Typically, individual subjects are given a randomized ID number, and their identifying data, such as name and address, are kept in a data file separate from the experimental data. If two data archivists are planning to allow a third party to perform a secondary analysis of an individual subject level join of their two databases, some method must be established to create a common randomized key.

One method that could be used for creating a common randomized key is private key encryption. The two data archivists could decide on a private encryption password. The commonly agreed upon ID of each subject could then be encrypted using the encryption password. This process would create a unique subject identifier for each subject so that, if the same subject appeared in each database, the unique identifier would be the same in each database. By using this method, the two data archivists would not need to reveal the identities of subjects to the secondary data analyst.

Another problem crops up as data is joined on the level of individual records. As more and more variables become present in a record, there is a greater and greater chance that a record is unique. Unique records that can be matched against publicly available data on individuals may allow for personal identification even though names and addresses have been removed from the file. This is not a problem with experimental data, but when experimental data becomes linked to enough demographic data, there is the chance that an individual can be identified.

There are methods to overcome this problem of the creation of unique records through the joining of databases (Dobra, Karr, Sanil, \& Fienberg, 2002). One method, called jittering (Willenborg \& de Wall, 2001), involves adding a small, normally distributed random value with mean of zero to all fields that could be used to identify an individual by matching against publicly accessible records. This method adds only a small amount of uncertainty to summary statistics and variance/covariance 
analyses but prevents direct matching of records to identify individuals.

\section{METADATABASES}

As the information from more and more data archives becomes available on the Web, searching and indexing programs such as Google will become increasingly useful as a method for organizing and cross referencing the metadata from individual data archives. The so-called harvesting of metadata from Web pages has become a focus of standards in order to increase the efficiency of Web search engines. The standards proposed by the Open Archives Initiative (http://www.openarchives.org) for metadata publication on Web pages provide a framework within which metadata can be optimized for harvesting so that scientific content can be efficiently linked.

The indexes that are created by these search engines will be able to be processed in a fashion similar to that which GenBank uses to measure similarities and associations in keywords and DNA sequences. One project that is using this sort of self-organizing map techniques is the WEBSOM Project at the Helsinki University of Technology (Kohonen et al., 2000; WEBSOM Project, 2003). This project organizes natural language text from Internet discussion groups into associational maps in which major topics and minor threads become self-organized into an associational structure.

By maintaining the metadata from scientific data archives on the Web in HTML format, it can be expected that a variety of strategies for the use and organization of these metadata will develop in the free-form, constantly changing environment of the Web. The potential for advance in the area of self-organizing data structures imposed on natural language is enormous and is rapidly developing, given the extraordinary amount of readily available raw material and the great need for its organization. No doubt, in the years to come, the current methods for searching for and obtaining data from the Web will seem antiquated and painfully labor intensive.

\section{A PRACTICAL IMPLEMENTATION}

What follows in this section is a recommended set of steps to place a node in a distributed data archive onto the Web. These steps are by no means comprehensive and are offered here as a recommendation by the authors in hopes of initiating community discussion about the form that a distributed archive might take. If a subset of the psychological research community comes to a consensus about this archive, we envision that an effort might be undertaken to write an open-source software package that would make the creation of metadata Web pages and their associated archives a task that would be self-explanatory for a graduate research assistant. Given the time constraints of an academic researcher, it seems unlikely that any other scenario would result in widespread adoption.

The recommended steps are the following.
1. Create an ASCII flat file (or files) of the data with randomized subject IDs and with all information removed that could identify an individual. If the data are images, use JPG format; if the data are audio, use MP3 format; and if the data are video, use Quicktime. Use the open-source version archiving software tar to bundle all of the data files into a single file. Use the open-source compression software gzip to compress the resulting file. Tar and gzip are available for free on all computing platforms, including Microsoft, Apple, Linux, and other Unix variants.

2. Create a publicly accessible metadata Web page as a homepage for this data set.

3. Include on this Web page the text that describes the methods by which the data were obtained, the meaning of the variables, the format of the data, and meaningful keywords. Use the standards for coding of metadata proposed by the Open Archives Initiative.

4. Include a bibliographic references section that links to full text versions (if possible) of any published articles in which these data are used. Include links to any other archive metadata Web pages that are related to these data.

5. Include any supplemental material that might have been removed prior to publication of a journal article, due to page limitations at the journal.

6. Code a single special keyword into the publicly accessible Web page: PsychologyDataArchive.

7. Create a password-protected page for the data file. The Web server administrator will need to do this, using commands appropriate to the server's operating system.

8. On the password-protected page, place a link to the data (either FTP or HTTP can be used).

9. Place an e-mail link on the public Web page so that the archivist can authorize access to the data via e-mail.

If these suggestions are followed, secondary analysts will be able to search with the keyword PsychologyDataArchive and locate all of the participating data archives with psychological data without being distracted by references to a subset of the billions of pages on the Web. This one keyword is enough to bind all of the participating Web sites into a single unit, a meta-archive that can be scanned by search engines. As this meta-archive grows and the search engines create self-organized maps of the keywords and links on the participating archives, a searchable crossreferenced virtual archive will emerge-one in which each of the participating scientists will still be able to maintain control over his or her own data.

\section{CONCLUSIONS}

This report has presented an overview of techniques that can be used to create usable data archives and store them into a heterogeneous distributed meta-archive on the Web. In such a distributed archive, individual researchers and their academic institutions retain responsibility for maintaining storage and managing access to the data. A major strength of a distributed archive in- 
cludes its heterogeneity, since it can grow and reorganize in an organic fashion as the capabilities of Web-based informational indexing software and intelligent agents consistently add semantic value to the meta-archive. One weakness of a distributed archive, in comparison with a centralized archive, is that one might envision data disappearing due to a junior faculty members' not receiving tenure or the death of a senior researcher. Although access control remains an issue in this case, perhaps taken on by the responsible academic institution, publicly accessible Web pages are constantly being cached and archived. One such project at http://www.archive.org is called the Way Back Machine and can show you snapshots of publicly accessible Web pages back to 1999. Thus, although access-controlled data may require some work to retrieve in 50 years, we expect that metadata pages in a distributed archive will remain easily accessible and will continue to add to the overall semantic network for the foreseeable future.

Several specific recommendations were made concerning some of the issues faced by the data archivist hoping to use the Web. The recommendations for making the data usable by the maximum number of secondary analysts are the following.

1. Store the data in an open-source and widely available format to facilitate future migration of the data to new platforms and operating systems.

2. Store the metadata in HTML format as a publicly accessible Web homepage for the data archive.

3. Place the keyword PsychologyDataArchive onto the publicly accessible Web homepage for the data archive and all other Web pages that search engines need to be able to access as part of the data archive.

4. Create a password-protected Web page to contain the data and then create an e-mail link on the homepage so that potential secondary analysts can easily e-mail for permission to use the data.

5. Provide access so that the entire data file can be downloaded as a single compressed file.

This article has proposed a distributed solution to some of the problems associated with behavioral science data archiving. It is unlikely that any one archiving solution will meet all of the needs of the behavioral science community, and we hope that there will be spirited discussion and development of ideas for archiving - including centralized scientific society sponsored solutions, as well as distributed open-source solutions that rely on self-assembled communities of interested parties. Out of this marketplace of ideas, we expect that some commonly agreed upon archiving mechanisms will emerge over the next decade - mechanisms that are likely to enhance the speed of scientific discovery in the behavioral sciences.

\section{REFERENCES}

Dobra, A., Karr, A. F., Sanil, A. P., \& Fienberg, S. E. (2002). Software systems for tabular data releases. International Journal of Uncertainty, Fuzziness \& Knowledge Based Systems, 10, 529-544.

GenBANK (1997). National Center for Biotechnology Information. Retrieved December 28, 2003 from http://www.ncbi.nlm.nih.gov/ Genbank/GenbankOverview.html.

IhaKa, R., \& Gentleman, R. (1996). R: A language for data analysis and graphics. Journal of Computational \& Graphical Statistics, 5 , 299-314.

InTERnet Software CONSORTIUm (2003). Internet domain survey. Retrieved December 28, 2003 from http://www.isc.org/.

Kohonen, T., Kaski, S., Lagus, K., Salojärvi, J., Honkela, J., PaAtero, V., \& SaArela, A. (2000). Self organization of a massive document collection. IEEE Transactions on Neural Networks, 11, 574-585.

Koutarou, D. K., Tissenbaum, H. A., Liu, Y., \& Ruvkun, G. (1997). $d a f-2$, an insulin receptor-like gene that regulates longevity and diapause in Caenorhabditis elegans. Science, 277, 942-946.

Kurzman, H. S., Church, R. M., \& Crystal, J. D. (2002). Data archiving for animal cognition research: Report of an NIMH workshop. Animal Learning \& Cognition, 30, 405-412.

Marsh, H. W., \& GraYson, D. (1995). Latent-variable models of multitrait-multimethod data. In R. H. Hoyle (Ed.), Structural equation modeling: Issues and applications (pp. 177-198). Newbury, CA: Sage.

McArdee, J. J., \& Hamagami, F. (1992). Modeling incomplete longitudinal and cross-sectional data using latent growth structural models. Experimental Aging Research, 18, 145-166.

McArdle, J. J., \& Horn, J. L. (1985). Mega analyses of the WAIS: Structural and dynamic models of adult intellectual ability (National Institute of Aging Grant report). Charlottesville: University of Virginia, Department of Psychology.

Netcraft (2003). Netcraft Web Survey. Retrieved December 28, 2003 from http://www.netcraft.com/Survey/Reports/.

RAFAELI, S., \& Tractinsky, N. (1991). Time in computerized tests: A multitrait, multimethod investigation of general knowledge and mathematical reasoning on-line examinations. Computers in Human Behavior, 7, 215-225.

Sullivan, D. (2003). Search engine sizes. Retrieved December 28, 2003 from http://www.searchenginewatch.com/reports/article.php/ 2156481 .

WEBSOM ProJect (2003). WEBSOM Project (Helsinki University of Technology). Retrieved December 28, 2003 from http://websom.hut.fi/ websom/.

WiLlENBORG, L. C. R. J., \& DE WALL, T. (2001). Elements of statistical disclosure control. New York: Springer-Verlag.

(Manuscript received December 30, 2003; revision accepted for publication July 13, 2004.) 